\title{
Gaining Mechanistic Insights into Cell Therapy Using Magnetic Resonance Imaging
}

\author{
Michel Modo ${ }^{1,2,3,4}$
}

Published online: 7 July 2016

(C) Springer International Publishing AG 2016

\begin{abstract}
Cell therapy remains a promising approach to treat neurological disorders with evidence of clinical efficacy emerging. However, the mechanisms underlying recovery and the conditions to achieve therapeutic success are poorly understood. Uncovering putative mechanisms and their interaction is essential to progress cell therapy from an interesting and promising possibility to a robust and consistent treatment that can be used to treat large cohorts of patients. Although there is a heavy focus on stem cell biology, relatively little effort has been dedicated to understanding the in vivo mechanisms that underlie efficacy. In vivo imaging and the development of appropriate biomarkers will be essential to gain a better mechanistic understanding of cell therapy in the treatment of neurological disease. The availability of magnetic resonance imaging in a clinical setting and its use in animal models allow for its use as a unique platform for mechanistic studies of cell therapy in a translational context.
\end{abstract}

Keywords MRI $\cdot$ Cell therapy $\cdot$ Mechanism $\cdot$ Neural stem cells $\cdot$ Bone marrow stem cell $\cdot$ Imaging

This article is part of the Topical Collection on Stem Cell Switches and Regulators

Michel Modo

modomm@upmc.edu

1 Department of Radiology, University of Pittsburgh, Pittsburgh, PA, USA

2 McGowan Institute for Regenerative Medicine, University of Pittsburgh, 3025 East Carson St, Pittsburgh, PA 15203, USA

3 Department of Bioengineering, University of Pittsburgh, Pittsburgh, PA, USA

4 Centre for the Neural Basis of Cognition, University of Pittsburgh, Pittsburgh, PA, USA

\section{Introduction}

The promise of cell therapy as an efficacious treatment for neurological conditions not only is contingent upon a thorough understanding of stem cell biology, but also requires optimization of the preparation and delivery of these cells and a deeper understanding of their interaction with the disease-damaged brain $[1,2]$. Although a variety of cell sources have been identified from developing, as well as adult tissues [3], three physiological processes have been proposed as key mechanisms for cell therapy to promote improvements in behavioral impairments in neurological conditions: (1) integration of implanted cells to replace lost or damaged cells, (2) supply of paracrine or juxtacrine factors leading to improvements in host tissue function, and/or (3) induction of host (e.g., inflammatory, neurogenesis) responses that lead to changes in the host tissue facilitating recovery. Considering the varied and synergistic effects of these processes that can be induced by implanted cells, it is extremely challenging to pinpoint precise processes as being the pivotal mechanisms leading to recovery [4]. Indeed, no one factor may be sufficient for recovery; rather, a cascade of interactions may be necessary to induce physiological changes that yield functional improvements [5]. As the brain consists of highly specialized regions, a further key component is the spatial sphere of action of these physiological processes. This can be very localized, as in the case of cell integration into existing functional or dysfunctional circuitry [6], or very diffuse, as in the case of a systemic factor release that affects the entire brain [7].

Considering that the various physiological processes require a spatial and temporal monitoring, it becomes evident that non-invasive in vivo serial monitoring can provide unique insights into the mechanisms involved in recovery. Indeed, cell therapy in a clinical setting is likely to require the use of diagnostic imaging to define patients who will benefit from 
the intervention and also to guide and monitor the effects of cell therapy. Clinical imaging techniques, such as computer tomography (CT), positron emission tomography (PET), and magnetic resonance imaging (MRI), have all been incorporated in early translational trials [8]. For instance, PET imaging of dopaminergic receptor binding has been crucial to demonstrate the functional activity of fetal tissue transplants in animal models [9], as well as patients with Parkinson's disease [10]. Although the ultimate goal is to use imaging to gain a mechanistic understanding of cell therapy in patients, this needs to be rooted in a thorough validation of imaging biomarkers that requires preclinical animal studies. Moreover, these animal studies provide the testing grounds to develop new approaches that could yield mechanistic insights.

\section{Location, Location, Location-Monitoring Cell Delivery and Distribution}

For cells to exert their physiological action, their location is pivotal. For instance, neural stem cells implanted into the periinfarct parenchyma of a stroke promote recovery, whereas cells injected into the lateral ventricle did not [11]. Considering the varied extent and topology of lesions caused by neurological conditions, such as stroke and traumatic brain injury, guidance of injection to the parenchyma is, hence, a crucial step to ensure that the transplanted cells can be efficacious [12]. Nevertheless, in other cases, direct targeting of the lesion cavity is important and MRI can provide the required guidance in terms of volume and location to perform a safe and efficient injection [13, 14]. Although most preclinical studies in rodents use a predefined implantation site irrespective of lesion size and location, neurosurgical planning is an essential component for other species, such as non-human primates [15], pig [16], and, of course, humans [17]. MR imaging can also be used to assess potential iatrogenic complications, such as injection tract damage [11] or the formation of cerebral microemboli after intra-arterial (i.a.) infusion of cells [18]. Real-time monitoring of the delivery of cells has been described by tagging cells with MRI contrast agents prior to injection [19]. This approach can also afford a determination of the accuracy of cell placement using cellular MRI [20], although consideration has to be given to the potential biological effects of the contrast agents that could potentially interfere with the cells' efficacy [21]. Co-injection of contrast agent in the infusate could, however, serve as a short-lived substitute to monitor delivery [22]. As the brain is a highly specialized organ and the efficacy of cells is likely due to their interaction with local tissue environments, defining the anatomical site, as well as the tissue characteristics of the environment, will likely be important to gain a mechanistic understanding of the cells' physiological action and will likely form the basis for future studies.
The characterization of the microenvironment can be achieved using advanced MRI techniques, such as diffusion MR histology and microtractography [23], as well as blood flow measurements and microvasculature [24]. However, in contrast, to define the determinants of the transplanted cell therapeutic efficacy, their distribution within the tissue and their sphere of spatial activity require serial monitoring. As cell suspensions permeate seamlessly into the existing parenchyma, visualization of the distribution of implanted cells and their migration requires the use of contrast agents to provide a surrogate marker of the cells. The preferred contrast agent for "cell tracking" by MRI is iron oxide, as it can provide a robust signal that can be detected against most tissue backgrounds for over 1 year [25]. This approach has found some clinical translation to assess the migration of neural progenitors after intraparenchymal administration into a patient with traumatic brain injury [26], as well as umbilical cord blood-derived stem cells upon intra-cerebroventricular injection into a patient with global cerebral ischemia [27]. However, small bleeds and air can induce an equivalent hypointense signal as that caused by iron oxide agents and lead to false positives. T1 agents could potentially produce an unequivocal signal for measuring the distribution of transplanted cells, but there can be significant quenching of this signal, which would prevent its detection [28]. Re-designing of contrast agents can overcome some of these shortcomings and provide a means for a robust and unequivocal mapping of transplanted cells within damaged brain tissue (Fig. 1) [29].

A different strategy for the detection of transplanted cells is to use multi-nuclei MRI and exploit the ${ }^{19} \mathrm{~F}$ signal to visualize implanted cells [30]. However, fluorine imaging is inherently low signal and is, hence, best suited to report on the distribution of large number of cells, as in the case of tissue engineering [31]. For tissue engineering in which multiple cell types might be implanted, differential contrast is needed to distinguish not only cells from the tissue background, but also each fraction of cells from one another. T1 and T2 imaging can be used for this purpose [32], but more selective imaging can be achieved using paramagnetic chemical exchange saturation transfer contrast agents (paraCEST) [33•]. In one study, paraCEST imaging afforded the visualization of the selective distribution of both neural stem cells (NSCs) and endothelial cells within a stroke cavity (Fig. 2). It can hence potentially be used to assess the dynamics of tissue morphogenesis. However, for i.a. or intra-venous (i.v.) cell injection, wholebody cell distribution will be required to determine the circulation time of cells, as well as their accumulation in different organs, such as the brain, lung, or spleen. MRI is not well suited to this type of analysis, whereas single-photon emission tomography (SPECT) achieves a high sensitivity that, in small rodents, is sufficient for whole-body imaging and revealed very few cells being retained in the brain after i.v. infusion, with a preferential localization to internal organs $[34,35]$. 

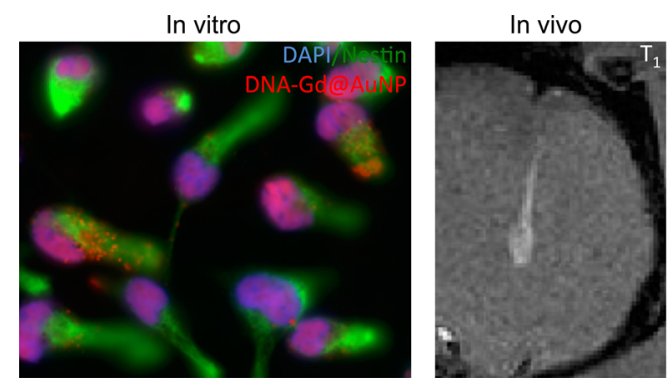

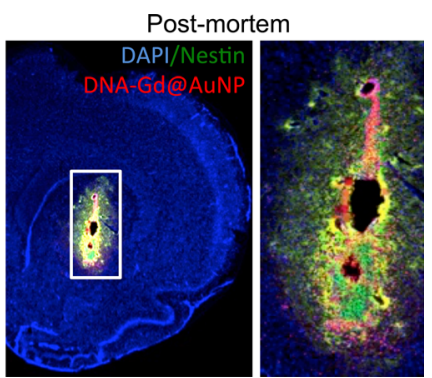

Fig. 1 MR imaging of intra-cerebrally transplanted NSCs. An MRI contrast agent consisting of DNA strands with gadolinium $\left(\mathrm{Gd}^{3+}\right)$ attached to gold $(\mathrm{Au})$ nanoparticles (DNA-Gd@AuNP) efficiently labels cells in vitro (red fluorescent tag) and affords the in vivo MR imaging of the distribution of implanted cells with histological validation and assessment of detection accuracy. This approach can be used to interrogate the relationship of cell position in relation to the tissue microenvironment to provide an indication as to which location of cells is important for therapeutic efficacy (adapted from [29])

therefore provides a unique means to assess the presence of implanted cells in a given tissue. Survival of cells, however, is not merely a function of "seeing" implanted cells tagged with a MRI contrast agent, as these can persist after cell death [37]. It has been suggested that surviving cells will yield a dilution and gradual attenuation of the signal due to proliferation and migration within the tissue, whereas contrast agent from dead cells will be more clustered around the injection site [38]. An alternative approach is to use MR-based nanosensors that, for instance, report on local $\mathrm{pH}$ changes surrounding transplanted cells to report on their viability [39]. However, interpretation of these signals is complicated and reliance on an additional imaging modality, such as bioluminescence, which only produces a signal for live cells is advocated to further develop cell vitality-based MR imaging [40]. Indeed, bioluminescence currently provides the gold standard for the non-invasive in vivo assessment of cell survival [41].

Beyond cell survival, the phenotypic differentiation of cells is key for some stem cells to exert their physiological functions within a tissue (e.g., neural stem cells function to produce differentiated neuronal cells). Evidence from fetal tissue invasive monitoring of the survival or rejection of cells

In vivo

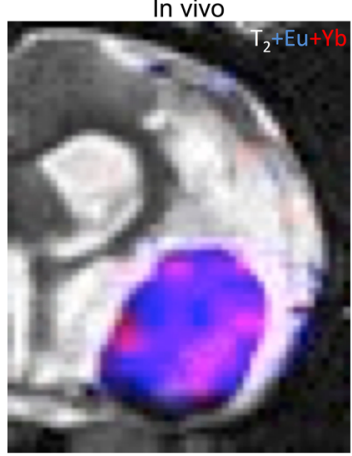

Post-mortem

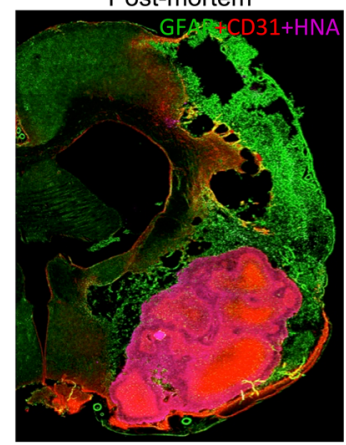

NSCs vs. ECs

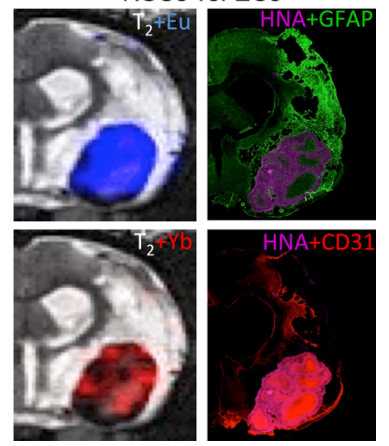

Fig. 2 MR imaging of in situ tissue engineering. To visualize the distribution of neural stem cells (NSCs) and endothelial cells (ECs), an imaging strategy that can afford the distinction of multiple signals needs to be employed. For this, paramagnetic chemical exchange saturation transfer (paraCEST) agents using Europium $(\mathrm{Eu})$ and Ybtterium $(\mathrm{Yb})$ were used to label NSCs and ECs, respectively, to afford the visualization of their in vivo distribution within a stroke cavity. Postmortem histology validated the distribution of these two populations of transplanted cells with single channel comparisons highlighting the selective detection of either cell type. This approach can be used in the context of tissue engineering to determine tissue morphogenesis and the interaction between multiple cell types (adapted from [33•]) 
transplantation in Parkinson's disease shows that dyskinetic side effects can be due to the ratio of serotonergic neurons within the dopaminergic transplant [42], highlighting the importance of achieving appropriate differentiation of transplanted cells. The in vivo assessment of cell phenotypes remains, nevertheless, a major challenge for MR imaging and is also reliant on using specific markers. At a more general level, the ability to distinguish between undifferentiated and differentiated cells can be considered. Undifferentiated cells typically proliferate, which leads to a higher metabolic activity, but also certain specific metabolites being present. To this end, MR spectroscopy can be used to distinguish between undifferentiated and differentiated cells based on elevated levels of phosphocholine + glycerophosphocholine, as well as myo-inositol [43]. However, MR spectroscopy does not readily lend itself to imaging, i.e., the ability to localize signals to a small area of tissue with a small number of cells, although it is suitable to characterize large tissue grafts in patients [44]. Thymidine analogs for PET imaging have been described to measure cell proliferation [45], and there is some effort to achieve a similar visualization using MRI [46], but so far, no study has applied these approaches to the imaging of cell therapy. Contrast agent-based approaches have been described to assess the functional status of transplanted cells [47], as well as their differentiation into inhibitory GABAergic neurons [48]. Although reliable cell surface imaging (e.g., receptors) for specific cell types is available using PET imaging [49], the poor spatial resolution and the inability to distinguish transplanted from host cells still limit its use to monitor differentiation of transplanted cells. However, in the case of Parkinson's disease, where dopaminergic cells are placed ectopically in the striatum, it is well suited to provide a macroscopic assessment of dopamine replacement in this location through the differentiation of cells into dopaminergic neurons [9]. At present, therefore, there is a dramatic lack of in vivo tools to directly visualize the differentiation of transplanted cells.

\section{Determining Functional Effects and Circuit Integration}

As in the case of cell differentiation into dopaminergic cells in Parkinson's disease, one aspect is to visualize the differentiation of cells using appropriate surrogate markers; the other is to demonstrate functional effects. In the case of Parkinson's, administration of a ligand acting on the dopamine system can be used to demonstrate that functional activity in the striatum has been re-established. Using amphetamine, a functional effect of fetal tissue grafts was demonstrated in a rat model of Parkinson's disease using pharmacological MRI (phMRI) [9] and the same principle was used to interrogate if NSC implantation preserved function through neuroprotection (Fig. 3) [50]. A functional integration of fetal tissue transplants

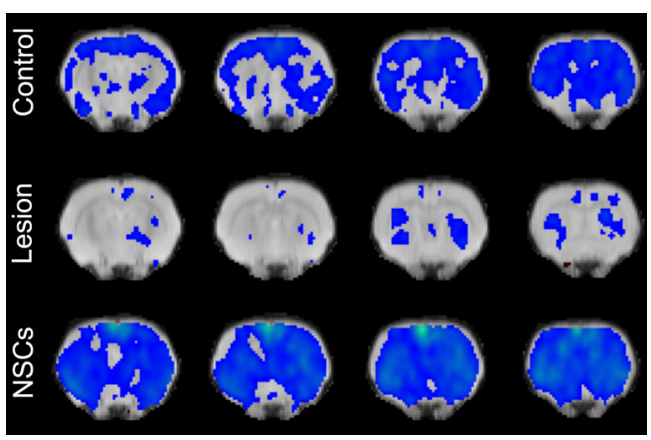

Fig. 3 Monitoring preservation of function using pharmacological MRI. Implantation of NSCs into a progressive pathological condition, such as Huntington's disease, revealed a spared functional response of tissue after administration of a D2 receptor agonist, indicating the cells' potential for neuropreservation. The use of functional or pharmacological MRI can provide unique insights into brain activity changes and functional connectivity due to cell therapy in the living brain that cannot be captured in histological studies (adapted from [50])

into the brain was demonstrated by functional MRI (fMRI). In patients with Huntington's disease, motor activity produced a functional input to grafted fetal tissue, which responded by producing neuronal activity that was detected on fMRI [51]. Using fMRI, NSCs have also been shown to alter brain plasticity after a stroke to promote recovery in the absence of significant neuroprotection or tissue replacement in animal models [52], as well as patients [53], further highlighting the unique potential of MRI to assess functional effects of cell therapy. Functional integration, therefore, can range from replacement of a critical signaling molecule, such as dopamine, to the formation of specific synapses within a neural circuitry.

A major advance to probe for circuit integration of transplanted cells has been achieved through the combination of optogenetics and fMRI (ofMRI). This approach takes advantage of being able to specifically activate cells containing optical actuators that are genetically encoded (e.g., channelrhodopsin). If only transplanted cells contain this gene, light can selectively stimulate transplanted cells to produce neuronal activity. Using fMRI, in contrast, can measure the response to this stimulus across the whole brain to indicate which areas are being activated by the optogenetic stimulus. Unlike the fMRI of activity in the fetal transplants, ofMRI affords probing of the downstream connectivity of implanted cells [54・*], hence, the effect of transplanted cells on host signal processing. An improvement of this approach over electrophysiology is that localization of implanted cells for recordings through an electrode is very challenging, and it is difficult to determine where the downstream connections are formed, whereas optogenetics can stimulate all relevant transplanted cells within a region of interest and record the global downstream effects using fMRI. Nevertheless, one has to be cautious and question as to how many cells 
will need to form connections within a given region to yield of a sufficient fMRI signal.

A further question that arises with implanted cells is their anatomical connectivity, which provides the substrate for functional connectivity within and across brain regions. Conventional histological methods are rather poor at highlighting this type of connectivity, but diffusion tensor imaging (DTI) is widely used clinically, as well as preclinically in animal models, to assess white matter connectivity and integrity. This approach has, for instance, been used in patients with Pelizaeus-Merzbacher disease to demonstrate remyelination by implanted NSCs, which differentiated into oligodendrocytes, hence, providing a putative biomarker in this phase I trial to assess efficacy in subsequent trials [55]. An increase in brain connectivity using DTI was also evidenced after autologous or allogeneic umbilical cord blood transfusions in children with cerebral palsy $[56 \bullet, 57]$. Yet, no clear mechanism of this change was determined. At present, this approach has found little use in larger trials of adult morbidities or preclinical studies, but a recent study highlighted its potential to uncover tissue integrity changes after endothelial progenitor cell infusion in mice subjected to stroke [58]. Although mostly applied to visualize fiber tracts of major white matter in the brain, DTI has the potential to interrogate tissue microstructure, including gray matter [59]. It is therefore widely underused as a "histological technique" to determine change in tissue characteristics due to pathology, as well as therapy. With sufficient spatial resolution, it can nevertheless afford a very detailed investigation of connectivity at the mesoscale ( $\mu \mathrm{m}$ to $\mathrm{mm}$ ) level (Fig. 4) [23] to characterize the tissue microenvironment in pathological, as well as regenerative, conditions.

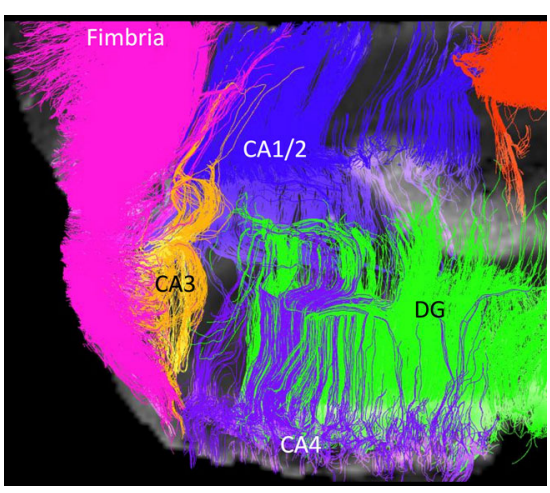

Fig. 4 MR histology and microtractography. DTI can trace fiber tracts that indicate inter- and intra-regional connectivity, as demonstrated here at an isotropic scale of $100 \mu \mathrm{m}$ in a human hippocampus biopsy. This approach can reveal changes in connectivity due to transplanted cells, either by indicating sparing of connections or by the re-establishment of appropriate connections (adapted from [23])

\section{Conclusion}

Uncovering putative mechanisms and their interaction is essential to progress cell therapy from an interesting and promising innovation to a robust and consistent treatment. Nevertheless, considering the varied modes of action of cell therapy, as well as its interaction and responsiveness to the tissue environment over time, histological studies are insufficient to investigate putative mechanisms. Non-invasive in vivo imaging techniques constitute an essential complementary line of analysis. Specifically, the biodistribution of cells within a treated organ or the whole organism, their migration, and survival within regions of damage, in addition to their impact on local and distant tissue function, are all pivotal aspects of their mode of action that will be crucial to guarantee the success of cell therapy in the clinic. A greater emphasis on in vivo imaging is, hence, required with a specific focus on uncovering the sufficient and necessary conditions for cell therapy to be efficacious.

Acknowledgments MM gratefully acknowledges funding by the National Institute for Neurological Disease and Stroke (R01NS08226; R21NS088167) and National Institute for Biomedical Imaging and Bioengineering (R01EB016629).

\section{Compliance with Ethical Standards}

Conflict of Interest Michel Modo declares that he has no conflict of interest.

Human and Animal Rights and Informed Consent This article does not contain any studies with human or animal subjects performed by any of the authors.

\section{References}

Papers of particular interest, published recently, have been highlighted as:

- Of importance

•- Of major importance

1. Rossetti T, Nicholls F, Modo M. Intracerebral cell implantation: preparation and characterization of cell suspensions. Cell Transplant. 2016;25(4):645-64. doi:10.3727/096368915X690350.

2. Lipsitz YY, Timmins NE, Zandstra PW. Quality cell therapy manufacturing by design. Nat Biotechnol. 2016;34(4):393-400. doi:10.1038/nbt.3525.

3. Trounson A, McDonald C. Stem cell therapies in clinical trials: progress and challenges. Cell Stem Cell. 2015;17(1):11-22. doi:10.1016/j.stem.2015.06.007.

4. Kalladka D, Muir KW. Brain repair: cell therapy in stroke. Stem Cells Cloning. 2014;7:31-44. doi:10.2147/SCCAA.S38003.

5. Chou $\mathrm{CH}$, Modo M. Human neural stem cell-induced endothelial morphogenesis requires autocrine/paracrine and juxtacrine signaling. Sci Rep. 2016;6:29029. doi:10.1038/srep29029. 
6. Thompson LH, Bjorklund A. Reconstruction of brain circuitry by neural transplants generated from pluripotent stem cells. Neurobiol Dis. 2015;79:28-40. doi:10.1016/j.nbd.2015.04.003.

7. Seifert HA, Leonardo CC, Hall AA, Rowe DD, Collier LA, Benkovic SA, et al. The spleen contributes to stroke induced neurodegeneration through interferon gamma signaling. Metab Brain Dis. 2012;27(2):131-41. doi:10.1007/s11011-012-9283-0.

8. Naumova AV, Modo M, Moore A, Murry CE, Frank JA. Clinical imaging in regenerative medicine. Nat Biotechnol. 2014;32(8): 804-18. doi:10.1038/nbt.2993.

9. Chen YI, Brownell AL, Galpern W, Isacson O, Bogdanov M, Beal $\mathrm{MF}$, et al. Detection of dopaminergic cell loss and neural transplantation using pharmacological MRI, PET and behavioral assessment. Neuroreport. 1999;10(14):2881-6.

10. Cochen V, Ribeiro MJ, Nguyen JP, Gurruchaga JM, Villafane G, Loc'h C, et al. Transplantation in Parkinson's disease: PET changes correlate with the amount of grafted tissue. Mov Disord. 2003;18(8):928-32. doi:10.1002/mds. 10463.

11. Smith EJ, Stroemer RP, Gorenkova N, Nakajima M, Crum WR, Tang E, et al. Implantation site and lesion topology determine efficacy of a human neural stem cell line in a rat model of chronic stroke. Stem Cells. 2012;30(4):785-96. doi:10.1002/stem.1024.

12. Janowski M, Engels C, Gorelik M, Lyczek A, Bernard S, Bulte JW, et al. Survival of neural progenitors allografted into the CNS of immunocompetent recipients is highly dependent on transplantation site. Cell Transplant. 2014;23(2):253-62. doi:10.3727 /096368912X661328.

13. Bible E, Chau DY, Alexander MR, Price J, Shakesheff KM, Modo M. Attachment of stem cells to scaffold particles for intra-cerebral transplantation. Nat Protoc. 2009;4(10):1440-53. doi:10.1038 /nprot.2009.156.

14. Massensini AR, Ghuman H, Saldin LT, Medberry CJ, Keane TJ, Nicholls FJ, et al. Concentration-dependent rheological properties of ECM hydrogel for intracerebral delivery to a stroke cavity. Acta Biomater. 2015;27:116-30. doi:10.1016/j.actbio.2015.08.040.

15. Bankiewicz KS, Bringas J, Pivirotto P, Kutzscher E, Nagy D, Emborg ME. Technique for bilateral intracranial implantation of cells in monkeys using an automated delivery system. Cell Transplant. 2000;9(5):595-607.

16. Silvestrini MT, Yin D, Martin AJ, Coppes VG, Mann P, Larson PS, et al. Interventional magnetic resonance imaging-guided cell transplantation into the brain with radially branched deployment. Mol Ther. 2015;23(1):119-29. doi:10.1038/mt.2014.155.

17. Watts C, Donovan T, Gillard JH, Antoun NM, Burnstein R, Menon DK, et al. Evaluation of an MRI-based protocol for cell implantation in four patients with Huntington's disease. Cell Transplant. 2003;12(7):697-704.

18. Cui LL, Kerkela E, Bakreen A, Nitzsche F, Andrzejewska A, Nowakowski A, et al. The cerebral embolism evoked by intraarterial delivery of allogeneic bone marrow mesenchymal stem cells in rats is related to cell dose and infusion velocity. Stem Cell Res Ther. 2015;6:11. doi:10.1186/scrt544.

19. Dick AJ, Guttman MA, Raman VK, Peters DC, Pessanha BS, Hill JM, et al. Magnetic resonance fluoroscopy allows targeted delivery of mesenchymal stem cells to infarct borders in Swine. Circulation. 2003;108(23):2899-904. doi:10.1161/01.CIR.0000095790.28368.F9.

20. Modo M, Hoehn M, Bulte JW. Cellular MR imaging. Mol Imaging. 2005;4(3):143-64.

21. Modo M, Kolosnjaj-Tabi J, Nicholls F, Ling W, Wilhelm C, Debarge $\mathrm{O}$, et al. Considerations for the clinical use of contrast agents for cellular MRI in regenerative medicine. Contrast Media Mol Imaging. 2013;8(6):439-55. doi:10.1002/cmmi.1547.

22. Su X, Kells AP, Salegio EA, Richardson RM, Hadaczek P, Beyer J, et al. Real-time MR imaging with Gadoteridol predicts distribution of transgenes after convection-enhanced delivery of AAV2 vectors. Mol Ther. 2010;18(8):1490-5. doi:10.1038/mt.2010.114.

23. Modo M, Hitchens TK, Liu JR, Richardson RM. Detection of aberrant hippocampal mossy fiber connections: ex vivo mesoscale diffusion MRI and microtractography with histological validation in a patient with uncontrolled temporal lobe epilepsy. Hum Brain Mapp. 2016;37(2):780-95. doi:10.1002/hbm.23066.

24. Boehm-Sturm P, Farr TD, Adamczak J, Jikeli JF, Mengler L, Wiedermann D, et al. Vascular changes after stroke in the rat: a longitudinal study using optimized magnetic resonance imaging. Contrast Media Mol Imaging. 2013;8(5):383-92. doi:10.1002 /cmmi.1534.

25. Obenaus A, Dilmac N, Tone B, Tian HR, Hartman R, Digicaylioglu $\mathrm{M}$, et al. Long-term magnetic resonance imaging of stem cells in neonatal ischemic injury. Ann Neurol. 2011;69(2):282-91. doi:10.1002/ana.22168.

26. Zhu J, Zhou L, XingWu F. Tracking neural stem cells in patients with brain trauma. N Engl J Med. 2006;355(22):2376-8. doi:10.1056/NEJMc055304.

27. Janowski M, Walczak P, Kropiwnicki T, Jurkiewicz E, DomanskaJanik K, Bulte JW, et al. Long-term MRI cell tracking after intraventricular delivery in a patient with global cerebral ischemia and prospects for magnetic navigation of stem cells within the CSF. PLoS One. 2014;9(2), e97631. doi:10.1371/journal.pone.0097631.

28. Modo M, Cash D, Mellodew K, Williams SC, Fraser SE, Meade TJ, et al. Tracking transplanted stem cell migration using bifunctional, contrast agent-enhanced, magnetic resonance imaging. Neuroimage. 2002;17(2):803-11.

29. Nicholls FJ, Rotz MW, Ghuman H, MacRenaris KW, Meade TJ, Modo M. DNA-gadolinium-gold nanoparticles for in vivo T1 MR imaging of transplanted human neural stem cells. Biomaterials. 2016;77:291-306. doi:10.1016/j.biomaterials.2015.11.021.

30. Ruiz-Cabello J, Walczak P, Kedziorek DA, Chacko VP, Schmieder $\mathrm{AH}$, Wickline SA, et al. In vivo "hot spot" MR imaging of neural stem cells using fluorinated nanoparticles. Magn Reson Med. 2008;60(6):1506-11. doi:10.1002/mrm.21783.

31. Bible E, Dell'Acqua F, Solanky B, Balducci A, Crapo PM, Badylak $\mathrm{SF}$, et al. Non-invasive imaging of transplanted human neural stem cells and ECM scaffold remodeling in the stroke-damaged rat brain by (19)F- and diffusion-MRI. Biomaterials. 2012;33(10):2858-71. doi:10.1016/j.biomaterials.2011.12.033.

32. Gilad AA, Walczak P, McMahon MT, Na HB, Lee JH, An K, et al. MR tracking of transplanted cells with "positive contrast" using manganese oxide nanoparticles. Magn Reson Med. 2008;60(1):17. doi:10.1002/mrm.21622.

33. Nicholls FJ, Ling W, Ferrauto G, Aime S, Modo M. Simultaneous MR imaging for tissue engineering in a rat model of stroke. Sci Rep. 2015;5:14597. doi:10.1038/srep14597. First example of noninvasive imaging of neural stem cells and endothelial cells implanted into a stroke cavity to visualize their relative distribution in the same subject. This study demonstrated that with ever more advanced tissue engineering approaches, significant imaging developments are required to meet the need for in vivo monitoring in translational paradigms.

34. Lappalainen RS, Narkilahti S, Huhtala T, Liimatainen T, Suuronen T, Narvanen A, et al. The SPECT imaging shows the accumulation of neural progenitor cells into internal organs after systemic administration in middle cerebral artery occlusion rats. Neurosci Lett. 2008;440(3):246-50. doi:10.1016/j.neulet.2008.05.090.

35. Khabbal J, Kerkela E, Mitkari B, Raki M, Nystedt J, Mikkonen V, et al. Differential clearance of rat and human bone marrow-derived mesenchymal stem cells from the brain after intra-arterial infusion in rats. Cell Transplant. 2015;24(5):819-28. doi:10.3727 /096368914X679336.

36. Kim H, Walczak P, Muja N, Campanelli JT, Bulte JW. ICVtransplanted human glial precursor cells are short-lived yet exert 
immunomodulatory effects in mice with EAE. Glia. 2012;60(7): 1117-29. doi:10.1002/glia.22339.

37. Cianciaruso C, Pagani A, Martelli C, Bacigaluppi M, Squadrito ML, Lo Dico A, et al. Cellular magnetic resonance with iron oxide nanoparticles: long-term persistence of SPIO signal in the CNS after transplanted cell death. Nanomedicine (Lond). 2014;9(10): 1457-74. doi:10.2217/nnm.14.84.

38. Berman SC, Galpoththawela C, Gilad AA, Bulte JW, Walczak P. Long-term MR cell tracking of neural stem cells grafted in immunocompetent versus immunodeficient mice reveals distinct differences in contrast between live and dead cells. Magn Reson Med. 2011;65(2):564-74. doi:10.1002/mrm.22613.

39. Chan KW, Liu G, Song X, Kim H, Yu T, Arifin DR, et al. MRIdetectable $\mathrm{pH}$ nanosensors incorporated into hydrogels for in vivo sensing of transplanted-cell viability. Nat Mater. 2013;12(3):26875. doi:10.1038/nmat3525.

40. Tennstaedt A, Mastropietro A, Nelles M, Beyrau A, Hoehn M. In vivo fate imaging of intracerebral stem cell grafts in mouse brain. PLoS One. 2015;10(12), e0144262. doi:10.1371/journal. pone. 0144262 .

41. Boehm-Sturm P, Aswendt M, Minassian A, Michalk S, Mengler L, Adamczak J, et al. A multi-modality platform to image stem cell graft survival in the naive and stroke-damaged mouse brain. Biomaterials. 2014;35(7):2218-26. doi:10.1016/j. biomaterials.2013.11.085.

42. Politis M, Wu K, Loane C, Quinn NP, Brooks DJ, Rehncrona S, et al. Serotonergic neurons mediate dyskinesia side effects in Parkinson's patients with neural transplants. Sci Transl Med. 2010;2(38):38ra46. doi:10.1126/scitranslmed.3000976.

43. Chung YL, El Akabawy G, So PW, Solanky BS, Leach MO, Modo M. Profiling metabolite changes in the neuronal differentiation of human striatal neural stem cells using $1 \mathrm{H}$-magnetic resonance spectroscopy. Neuroreport. 2013;24(18):1035-40. doi:10.1097 /WNR.0000000000000056.

44. Ross BD, Hoang TQ, Bluml S, Dubowitz D, Kopyov OV, Jacques $\mathrm{DB}$, et al. In vivo magnetic resonance spectroscopy of human fetal neural transplants. NMR Biomed. 1999;12(4):221-36.

45. Bading JR, Shields AF. Imaging of cell proliferation: status and prospects. J Nucl Med. 2008;49 Suppl 2:64S-80. doi:10.2967 /jnumed.107.046391.

46. Bar-Shir A, Liu G, Liang Y, Yadav NN, McMahon MT, Walczak P, et al. Transforming thymidine into a magnetic resonance imaging probe for monitoring gene expression. J Am Chem Soc. 2013;135(4):1617-24. doi:10.1021/ja312353e.

47. Himmelreich U, Aime S, Hieronymus T, Justicia C, Uggeri $F$, Zenke $\mathrm{M}$, et al. A responsive MRI contrast agent to monitor functional cell status. Neuroimage. 2006;32(3):1142-9. doi:10.1016/j. neuroimage.2006.05.009.

48. Aswendt M, Gianolio E, Pariani G, Napolitano R, Fedeli F, Himmelreich $\mathrm{U}$, et al. In vivo imaging of inhibitory, GABAergic neurons by MRI. Neuroimage. 2012;62(3):1685-93. doi:10.1016/j. neuroimage.2012.05.029.

49. Sim N, Parker D. Critical design issues in the targeted molecular imaging of cell surface receptors. Chem Soc Rev. 2015;44(8): 2122-34. doi:10.1039/c4cs00364k.

50. Roberts TJ, Price J, Williams SC, Modo M. Pharmacological MRI of stem cell transplants in the 3-nitropropionic acid-damaged striatum. Neuroscience. 2007;144(1):100-9. doi:10.1016/j. neuroscience.2006.09.015.

51. Bluml S, Kopyov O, Jacques S, Ross BD. Activation of neurotransplants in humans. Exp Neurol. 1999;158(1):121-5. doi:10.1006/exnr.1999.7073.

52. Ramos-Cabrer P, Justicia C, Wiedermann D, Hoehn M. Stem cell mediation of functional recovery after stroke in the rat. PLoS One. 2010;5(9), e12779. doi:10.1371/journal.pone.0012779.

53. Bhasin A, Srivastava M, Bhatia R, Mohanty S, Kumaran S, Bose S. Autologous intravenous mononuclear stem cell therapy in chronic ischemic stroke. J Stem Cells Regen Med. 2012;8(3):181-9.

54.• Byers B, Lee HJ, Liu J, Weitz AJ, Lin P, Zhang P, et al. Direct in vivo assessment of human stem cell graft-host neural circuits. Neuroimage. $2015 ; 114: 328-37$. doi: $10.1016 / j$. neuroimage.2015.03.079. Mechanistic insights into neuronal connectivity are very challenging using conventional approaches. The combination of optogenetic and functional magnetic resonance imaging to interrogate the functional impact of transplanted cells provides a major step forward to assess cell replacement in neurological conditions.

55. Gupta N, Henry RG, Strober J, Kang SM, Lim DA, Bucci M, et al. Neural stem cell engraftment and myelination in the human brain. Sci Transl Med. 2012;4(155):155ra37. doi:10.1126 /scitranslmed.3004373.

56. Englander ZA, Sun J, Laura C, Mikati MA, Kurtzberg J, Song AW. Brain structural connectivity increases concurrent with functional improvement: evidence from diffusion tensor MRI in children with cerebral palsy during therapy. Neuroimage Clin. 2015;7:315-24. doi:10.1016/j.nicl.2015.01.002. Evidence that non-invasive imaging, such as diffusion tensor imaging, are key outcome measures to evaluate the efficacy of cell therapy in patients and that more preclinical studies are needed to validate these biomarkers

57. Min K, Song J, Kang JY, Ko J, Ryu JS, Kang MS, et al. Umbilical cord blood therapy potentiated with erythropoietin for children with cerebral palsy: a double-blind, randomized, placebo-controlled trial. Stem Cells. 2013;31(3):581-91. doi:10.1002/stem.1304.

58. Bai YY, Peng XG, Wang LS, Li ZH, Wang YC, Lu CQ, et al. Bone marrow endothelial progenitor cell transplantation after ischemic stroke: an investigation into its possible mechanism. CNS Neurosci Ther. 2015;21(11):877-86. doi:10.1111/cns.12447.

59. Basser PJ, Pierpaoli C. Microstructural and physiological features of tissues elucidated by quantitative-diffusion-tensor MRI. J Magn Reson B. 1996;111(3):209-19. 